\title{
Extension of Basic Linear Mixed Effect Model to Incorporate Heterocedasticity
}

\author{
Nageena Nazir $^{1 *}$, S.A. Mir ${ }^{1}$, Zahoor Ahmed ${ }^{2}$ and M. Iqbal Jeelani ${ }^{3}$ \\ ${ }^{1}$ Division of Agricultural Statistics, Sher-e-Kashmir University of Agricultural Sciences and \\ Technology, Kashmir, India \\ ${ }^{2}$ KVK Kupwara, Sher-e-Kashmir University of Agricultural Sciences and Technology, \\ Kashmir, India \\ ${ }^{3}$ Division of Agricultural Statistics, Sher-e-Kashmir University of Agricultural Sciences and \\ Technology, Jammu, India \\ *Corresponding author
}

\section{A B S T R A C T}

\begin{tabular}{|l|}
\hline Ke y w o r d s \\
$\begin{array}{l}\text { Extension, Basic linear } \\
\text { mixed effect model, } \\
\text { Heterocedasticity }\end{array}$ \\
\hline Article Info \\
\hline $\begin{array}{l}\text { Accepted: } \\
\text { 06 May } 2018 \\
\text { Available Online: } \\
\text { 10 June } 2018\end{array}$ \\
\hline
\end{tabular}

\section{Introduction}

The linear mixed effect model allows considerable flexibility in the specification of the random effects structure but restricts the within group errors to be independent, identically distributed random variables with mean zero and constant variance. This basic linear mixed effects model provides an adequate model for many different types of grouped data observed in practice. However, there are many applications involving grouped data for which the within group errors are heteroscedastic (i.e. have unequal variances), correlated or both. The lme() function of nlme library due to pinherio and bates 2000 is used to fit the extended linear mixed-effects model. The complexity of linear mixed-effects (LME) models means that traditional diagnostics are rendered less effective. This is due to a breakdown of asymptotic results, boundary issues, and visible patterns in residual plots that are introduced by the model fitting process. Loy et al., has proposed adjustment to some of the well-known issues (Loy, 2013). 
In this paper we extent the basic linear mixed effects model to allow heteroscedastic errors. We describe how the lme() function can be used to fit the extended linear mixed effects model and illustrate its various capabilities through examples. Also we will show the estimation and computational methods of simple linear mixed effect models can be applied to the extended model. To fit the extended model we have used a real Animal Science data set. The data generated from the experiment on chicks during 2012 by Division of LPT Skuast-Kashmir, Shuhama has been used for the present study. Data was collected on bodyweight of chicks measured over a period of 64 days. The bodyweight was measured on day 1 and every 7 days thereafter with an extra measurement on $44^{\text {th }}$ day. Thus in total eleven readings were collected from each chick. There were three groups of chicks on different diets with 5 chicks in each group. The data set was named Chick Weight for analysis and modeling in R/SAS software's. It has 165 rows and 4 columns. The columns names are weight, time, chick and diet for body weight of chicks, time interval, chick number and diet respectively.

\section{General formulation of extended linear} mixed effects model

In the linear mixed effects model given below:

$y_{i}=x_{i} \beta+z_{i} b_{i}+e_{i} i=1,2$,

$\mathrm{b}_{\mathrm{i}} \sim \mathrm{N}(\mathrm{o}, \Sigma), \mathrm{e}_{\mathrm{i}} \sim \mathrm{N}\left(0, \sigma^{2} I\right)$

It is assumed that the within group errors $\mathrm{e}_{\mathrm{i}}$ are independent $\mathrm{N}\left(0, \sigma^{2} I\right)$ random vectors. In the extended single level linear mixed effect model we assume heterocedastic and correlated within group errors, which can be expressed as:

$\mathrm{y}_{\mathrm{i}}=\mathrm{x}_{\mathrm{i}} \beta+\mathrm{z}_{\mathrm{i}} \mathrm{b}_{\mathrm{i}}+\mathrm{e}_{\mathrm{i}}$ $M(2.2)$ $\mathrm{b}_{\mathrm{i}} \sim \mathrm{N}(0, \Psi), \mathrm{e}_{\mathrm{i}} \sim \mathrm{N}\left(0, \sigma^{2} \Lambda_{i}\right) i=1,2$, ............. M

Where the $\Lambda_{\mathrm{i}}$ is positive definite matrices parameterized by a fixed generally small, set of parameters $\lambda$. As in basic LMEM (2.1) specified above, the within group $e_{i}$ are assumed to be independent for different $i$ and independent of random effect $b_{i}$. The $\sigma^{2}$ is factored out of the $\Lambda_{i}$ for computational reasons (It can then be eliminated from the profiled likelihood function).

\section{Estimation and computational methods}

Several methods of parameter estimation have been used for linear mixed effects models same will be used for extended linear mixed effect models. Among them the two general methods are ML and REML the detailed description of the two can be find from (Pinherio and Bates 2000, section 2.2). Because $\Lambda_{i}$ is positive definite, it admits an invertible square root $\Lambda_{i}^{1 / 2}$ (Thisted, 1988) with inverse $\Lambda_{i}^{-1 / 2}$ such that $\Lambda_{i}=\left(\Lambda_{i}^{1 / 2}\right)^{T} \Lambda_{i}^{1 / 2}$ and $\Lambda_{i}^{-1}$ $=\Lambda_{i}^{-1 / 2}\left(\Lambda_{i}^{-1 / 2}\right)^{T}$.

Letting $\mathrm{y}_{\mathrm{i}}^{*}=\left(\Lambda_{\mathrm{i}}^{-1 / 2}\right)^{\mathrm{T}} \mathrm{y}_{\mathrm{i}}, \mathrm{e}_{\mathrm{i}}^{*}=\left(\Lambda_{\mathrm{i}}^{-1 / 2}\right)^{\mathrm{T}} \mathrm{e}_{\mathrm{i}} \mathrm{x}_{\mathrm{i}}{ }^{*}=\left(\Lambda_{\mathrm{i}}^{-}\right.$ $\left.{ }^{1 / 2}\right)^{\mathrm{T}} \mathrm{x}_{\mathrm{i}}, \mathrm{Z}_{\mathrm{i}}^{*}=\left(\Lambda_{\mathrm{i}}^{-1 / 2}\right)^{\mathrm{T}} \mathrm{Z}_{\mathrm{i}}(2.3)$

and noting that $\mathrm{e}_{\mathrm{i}}^{*} \sim \mathrm{N}\left[\left(\Lambda_{\mathrm{i}}^{-1 / 2}\right)^{\mathrm{T}} 0, \sigma^{2}\left(\Lambda_{\mathrm{i}}^{-1 / 2}\right)^{\mathrm{T}}\right.$ $\left.\Lambda_{\mathrm{i}} \Lambda_{\mathrm{i}}^{-1 / 2}\right]=\mathrm{N}\left(0, \sigma^{2} I\right)$.

Thus the model 2.2 can be revived as

$y_{i}^{*}=\mathrm{x}_{\mathrm{i}}^{*} \beta+\mathrm{z}_{\mathrm{i}}^{*} \mathrm{~b}_{\mathrm{i}}+\mathrm{e}_{\mathrm{i}}^{*} i=1,2, \ldots \ldots \ldots \ldots . \mathrm{M}$ (2.4)

$\mathrm{b}_{\mathrm{i}} \sim \mathrm{N}(0, \Psi), \mathrm{e}_{\mathrm{i}}^{*} \sim \mathrm{N}\left(0, \sigma^{2} I\right) i=1,2$, $\mathrm{M}$

That is $y_{i}^{*}$ can be described by a basic linear mixed effects model. Since the differential of 
the linear transformation $\mathrm{y}_{\mathrm{i}}^{*}=\left(\Lambda_{\mathrm{i}}^{-1 / 2}\right)^{\mathrm{T}} \mathrm{y}_{\mathrm{i}}$ is simply $\mathrm{dy}_{\mathrm{i}}{ }^{*}=\left|\Lambda_{\mathrm{i}}^{-1 / 2}\right| \mathrm{dy}_{\mathrm{i}}$, the likelihood function, $\mathrm{L}$ corresponding to the extended linear mixed effects model (2.2) is the probability density for the data given the parameters, but regarded as a function of the parameters with data fixed instead of as a function of data with parameters fixed and can be expressed as

$$
L\left(\beta, \theta, \sigma^{2}, \lambda \mid y\right)=\prod_{i=1}^{M} p\left(y_{i} \mid \beta, \theta, \sigma^{2}, \lambda\right)
$$

Since the non-observable random vector $b_{\mathrm{i}} i=$ $1, \ldots, \mathrm{M}$ are part of the model, we must integrate the conditional density of the data given the random effect to obtain the marginal density for the data. We can use the independence of $b_{i}$ and $e_{i}$ to express this as

$$
\prod_{i=1}^{M} p\left(y_{i}^{*} \mid \beta, \theta, \sigma^{2}, \lambda\right) \mid \Lambda_{i}^{-1 / 2}=L\left(\beta, \theta, \sigma^{2}, \lambda \mid y^{*}\right)
$$

Where $\mathrm{P}$ (.) denotes a probability density function, the conditional density of $y_{i}$ and marginal density of $b_{i}$ both are multivariate normal. Since the likelihood $\mathrm{L}\left(\beta, \theta, \sigma^{2}\right.$, $\left.\lambda \mid y^{*}\right)$ corresponds to a basic linear mixed effects models and thus all the results of the LMEM (see PinHerio and Bates2000 section 2.2) applies to this as well. Thus the evaluation of the likelihood can be made by orthogonal triangular decomposition just like in case of simple linear mixed effects model leading to numerically efficient algorithm for maximum likelihood estimation.

Although technically the random effects $b_{i}$ are not parameters for the statistical model, they do behave in some ways like parameters often we want to estimate their values. The conditional models of the random effects evaluated at the conditional estimate of $\beta$ are best linear unbiased predictors or BLUP's of the $\mathrm{b}_{\mathrm{i}}, i=1,2$,--- M. They can be evaluated using the matrices from the orthogonal triangular decomposition. In practice the unknown vector $\theta_{i}$ is replaced by its maximum likelihood estimate $\hat{\theta}_{i}$, producing estimated BLUP's $\hat{b}_{i}\left(\theta_{i}\right)$.

There are several ways to define REML estimation criterion one definition that provides a convenient computational from (Laird and Ware, 1982) is:

$\mathrm{L}_{\mathrm{R}}\left(\theta, \sigma^{2} \mid \mathrm{y}\right)=\int L\left(\beta, \theta, \sigma^{2} \mid y\right) d \beta$

Which within Bayesian framework corresponds to assuming a locally uniform prior distribution for the fixed effects $\beta$ and integrating them out of the likelihood. $M$ Restricted likelihood corresponding to the Mxtended linear mixed effects models is $i=$ defined as in (4.7) above by integrating out the fixed effects from the likelihood.

$L_{R}\left(\theta, \sigma^{2}, \lambda \mid y\right)=\int L\left(\beta, \theta, \sigma^{2}, \lambda \mid y\right) d \beta=L_{R}\left(\theta, \sigma^{2}, \lambda \mid y^{y}\right) \prod \mid \Lambda_{i}^{-1 / 2}$

Since the function $L\left(\beta, \theta, \sigma^{2}, \lambda \mid y^{*}\right)$ corresponds to a restricted likelihood function of a basic linear mixed effects model. Hence the results of linear mixed effects models can be used to obtain a numerically efficient representation of the profiled log restricted likelihood (for details see of Pin Herio and Bates).

Just like the LME models the optimization of the $\log$ likelihood or restricted likelihood of extended linear mixed effects model can usually accomplished through EM algorithm (Dempster et al., 1977) or through Newton Raphsan iterations (Laird and Ware, 1982; Lindstram and Bates, 1988; Langford, 1993, Thisted, 1988, §4.2.2). 
Variance functions for modelling heterocedasticity

Variance functions are used to model the variance structure of the within group errors using covariates. They have been studied in detail in the context of linear mixed effects models by Davidian and Giltinan (1995). Following Davidian and Giltinan (1995, Ch. 4) we define the general variance function model for the within group errors in the extended single level linear mixed effects model (2.2) as:

$$
\operatorname{var}\left(e_{i j} \mid b_{i}\right)=\sigma^{2} g^{2}\left(\mu_{i j}, v_{i j}, \delta\right)_{i=1,----\mathrm{M} \mathrm{j}}
$$$$
=1,2---\mathrm{n} 3.1
$$

Where $\mu_{i j}=E\left[y_{i j} \mid b_{i}\right], \quad v_{i j}$ is a vector of variance covariates, $\delta$ is a vector of variance parameters and $\mathrm{g}($.$) is the variance function,$ assumed continuous in $\delta$. For example, if the within group variability is believed to increase with same power of absolute value of a covariate $\mathrm{v}_{\mathrm{ij}}$, we can write the variance model as: $\operatorname{var}\left(\mathrm{e}_{\mathrm{ij}} \mid \mathrm{b}_{\mathrm{i}}\right)=\sigma^{2}\left(\mathrm{v}_{\mathrm{ij}}\right)^{2 \delta}$. The variance function in this case is $g(x, y)=|x|^{y}$ and the covariate $\mathrm{v}_{\mathrm{ij}}$ can be the expected value $\mu_{\mathrm{ij}}$. The variance function formulation (3.1) is very flexible and inductive, because it allows the within group variance to depend on the fixed effects $\beta$, and the random effects $b_{i}$, through the expected values $\mu_{\mathrm{ij}}$. However as discussed in Davidian and Giltinan (1995 ch. 4), it poses some theoretical and computational difficulties as the within group errors and the random effects can no longer assumed to be independent. Under the assumption that $\mathrm{E}$ $\left[\mathrm{e}_{\mathrm{i}} \mid \mathrm{b}_{\mathrm{i}}\right]=0$, it is easy to verify that $\operatorname{var}\left(\mathrm{e}_{\mathrm{ij}}\right)=\mathrm{E}$ (var $\left(e_{i j} \mid b_{i}\right)$, so that the dependence on the unobserved random effects can be avoided by integrating them out of the variance mode. Because the variance function of $g$ is generally non-linear in $b_{i j}$ integrating the random effects out of the variance model (3.1) does not lead to a computationally feasible optimization procedure. Instead we proceed as in Davidian and Gillinan (1995, Ch. 6) and use an approximate variance model in which the expected value $\mathrm{u}_{\mathrm{ij}}$ are replaced by their BLUPs

$\hat{\mu}_{i j}=x_{i j}^{T} \beta+z_{i j}^{T} \hat{b}_{i}$

Where $x_{i j}$ and $z_{i j}$ denotes, respectively the $j^{\text {th }}$ row of $X_{i}$ and $Z_{i}$, thus

V

$\approx \sigma^{2} g^{2}\left(\hat{\mu}_{\mathrm{ij}}, \mathrm{V}_{\mathrm{ij}}, \delta\right) \quad \mathrm{i}=1,2----\mathrm{M}$

$j=1,2----\mathrm{n}_{\mathrm{i}}(3.2)$

Under this approximation, the within group error are assumed independent of the random effects as in 2.2 and the results in section 2.1 can still be used. Note that if the condition variance model (3.1) does not depend on $\mu_{\mathrm{ij}}$, (3.2) gives the exact marginal variance and no appropriation is required.

when the conditional variance model (3.1) depends on $\mu_{\mathrm{ij}}$, the optimization algorithm follows an "iteratively reweighted" scheme, for given $\beta^{(t)}, \theta^{\mathrm{t}}, \lambda^{\mathrm{t}}$, the corresponding BLUP's $\hat{\mu}_{\mathrm{ij}}{ }^{(\mathrm{t})}$ can be obtained and held fixed while the objective function is optimized to produce now estimates $\beta^{(t+1)}, Q^{(t+1)}, \lambda^{L+1}$ which in turn give updated BLUP's $\hat{\mu}_{\mathrm{ij}}{ }^{(t+1)}$ with the process iterating until convergence. The resulting estimates approximate the (restricted) maximum likelihood estimates. When the variance model does not involve $\mu_{\mathrm{ij}}$ the likelihood can be directly optimized producing the exact (restricted) maximum likelihood estimates

\section{Variance function in nlme}

The nlme library of R-software provides a set of classes of variance functions, the varFunc 
classes, that can be used to specify the within group variance models in the extended linear mixed effects model. Table 1 lists the standard varFunc classes induced in the nlme library. The varFunc constructors have the same name as their corresponding class.

The two main arguments for most of the varFunc constructors are value and form. The first specifies the value of the variance parameter $\delta$ and the second is a one-sided formula specifying the variance covariate $\mathrm{V}$ and optionally a stratification variable for the variance parameters - different parameters are used for each level of stratified variable.

\section{Using variance function with Ime()}

The above mentioned variance functions can be used in the $\operatorname{lme}()$ functions using the weights argument. By default, weights = NULL, corresponding to a homescedastic variance model for the within group errors. Variance models can be specified in weights either as a one-sided formula, in which case it is passed as the single argument to the varFixed constructor, or as a varFunc object, created using the standard constructors given in 3.1 .

To illustrate the use of variance functions with lme(), we use the Body Weight data set introduced earlier. The function grouped Data() of the nlme library of R-software and dotplot() function of lattice library were used for the graphical summary so as to understand the nature of the data. The plot method for the grouped Data class allows an optional argument outer that can be either a logical value or a formula. When this argument is used the panels are determined by the factor or combination of factors given in the outer formula. This provides a strong visual comparison of the difference between the levels of the factor. The graphic summary is reported below:
Figure 1 provides a comparison of difference between the diets for the bodyweight data set. It produces three panels for the three different diets. Each panel represents a scatter plot of the response versus a continuous variate. The points in the each group are joined by lines, where each line represents the weight of a particular chick over a period of 64 days.

It also indicates strong differences among the three diet groups. There is also evidence of a chick in diet group 2 with an unusually high initial body weight. The body weights appear to grow linearly with time, possibly with different intercepts and slopes for each diet, and with intercept and slope random effects to account for chick-to-chick variation. The dotplot of bodyweights of chicks measured over a period of 64 days is reported in Figure 2.

As can be seen from the figure the bodyweights varies with all the three diets, diet-3 shows the maximum gain in weight of chicks followed by diet-2. However in diet- 2 there is a chick with unusually high initial bodyweight.

We first fit a model using the lme() function of R-software were we assume homocedasticity i.e., we fit a homocedastic mixed effects model by using the argument weight=NULL in the $\operatorname{lme}()$ function the commands for all the functions used are given in the chapter one of the manuscript. The results obtained are summarised below.

The Table 2 provides the values of different fixed effects and their interaction values along with their standard error, t-cal and p-value. From the table it is clear that there appears to be significant increase in growth rate associated with Diet2 (TimeDiet2) and a boarder line significant increase in growth rate for Diet3 (TimeDiet3). We can further assess the variability in the parameter estimates with the intervals method. 
Table.1 Standard var-Func classes

\begin{tabular}{|l|l|}
\hline VarFixed & Fixed variance \\
\hline VarIdent & Different variance per stratum \\
\hline VarPower & Power of covariance \\
\hline VarExp & Exponential of covariates \\
\hline VarConstPower & constant plus power of covariates \\
\hline VarCanb & Combination of variance functions \\
\hline
\end{tabular}

Table.2 Summary of the results of the fixed effects obtained by fitting homocedastic mixed effects model

\begin{tabular}{|c|c|c|c|c|}
\hline & Coefficient & Standard Error & t value & p value \\
\hline (Intercept) & 251.65 & 13.09 & 19.22 & 0.0000 \\
\hline Time & 0.36 & 0.091 & 3.95 & 0.0001 \\
\hline Diet2 & 200.66 & 22.68 & 8.85 & 0.0000 \\
\hline Diet3 & 252.07 & 22.68 & 11.11 & 0.0000 \\
\hline Time:Diet2 & 0.61 & 0.16 & 3.84 & 0.0002 \\
\hline Time:Diet3 & 0.29 & 0.15 & 1.89 & 0.0606 \\
\hline
\end{tabular}

Table.3 95\% confidence intervals for fixed effects

\begin{tabular}{|c|c|c|c|}
\hline & Estimate & Lower & Upper \\
\hline (Intercept) & 251.65 & 225.78 & 277.51 \\
\hline Time & 0.36 & 0.179 & 0.54 \\
\hline Diet2 & 200.66 & 151.67 & 249.66 \\
\hline Diet3 & 252.07 & 203.08 & 301.07 \\
\hline Time:Diet2 & 0.06 & 0.29 & 0.092 \\
\hline Time:Diet3 & 0.29 & -0.01 & 0.61 \\
\hline
\end{tabular}

Table.4 95\% confidence intervals for random effects

\begin{tabular}{|l|c|c|c|} 
& Estimate & Lower & Upper \\
\hline Sd(intercept) & 36.94 & 25.10 & 54.36 \\
\hline sd(Time) & 0.248 & 0.16 & 0.37 \\
\hline cor((Inter),Time) & -0.149 & -0.61 & 0.39 \\
\hline Within group standard error & $\mathbf{4 . 4 4}$ & 3.95 & 4.98 \\
\hline
\end{tabular}

Table.5 Summary of the results of the fixed effects obtained by fitting varPower (Heterocedastic) mixed effects model

\begin{tabular}{|c|c|c|c|c|}
\hline & Value & Std. Error & t-value & p-value \\
\hline (Intercept) & 251.65 & 13.06 & 19.25 & 0.0000 \\
\hline Time & 0.36 & 0.09 & 4.08 & 0.0001 \\
\hline Diet2 & 200.66 & 22.65 & 8.86 & 0.0000 \\
\hline Diet3 & 252.07 & 22.66 & 11.12 & 0.0000 \\
\hline Time:Diet2 & 0.61 & 0.16 & 3.87 & 0.0002 \\
\hline Time:Diet3 & 0.29 & 0.15 & 1.89 & 0.0601 \\
\hline
\end{tabular}


Table.6 95\% confidence intervals for fixed effects

\begin{tabular}{|c|c|c|c|}
\hline & Estimate & Lower & Upper \\
\hline Intercept & 251.60 & 22.79 & 277.41 \\
\hline Time & 0.36 & 0.19 & 0.54 \\
\hline Diet2 & 200.77 & 151.83 & 249.72 \\
\hline Diet3 & 252.17 & 203.21 & 301.12 \\
\hline Time:Diet2 & 0.60 & 0.29 & 0.91 \\
\hline Time:Diet3 & 0.29 & -0.01 & 0.60 \\
\hline
\end{tabular}

Table. 7 95\% confidence intervals for random effects

\begin{tabular}{|l|c|c|c|}
\hline & Estimate & Lower & Upper \\
\hline Sd(intercept) & 36.89 & 25.05 & 54.33 \\
\hline sd(Time) & 0.24 & 0.16 & 0.37 \\
\hline cor((Inter),Time) & -0.15 & -0.62 & 0.40 \\
\hline Variance function Power & 0.54 & 0.21 & 0.88 \\
\hline Within Group Standard error & 0.18 & 0.024 & 0.13 \\
\hline
\end{tabular}

Table.8 Empirical comparison of the fitted models i.e. homocedastic mixed effects model and varPower (heterocedastic) mixed effects model

\begin{tabular}{|l|c|c|c|c|c|}
\multicolumn{1}{|c|}{ Models } & AIC & BIC & logLik & L. ratio test & p-value \\
\hline Homocedastic Model & 1171.72 & 1203.07 & -575.85 & & \\
\hline varPower Model & 1163.92 & 1198.41 & -570.96 & 9.80 & 0.0017 \\
\hline
\end{tabular}

Fig.1 Weight of chicks versus time for three different diets

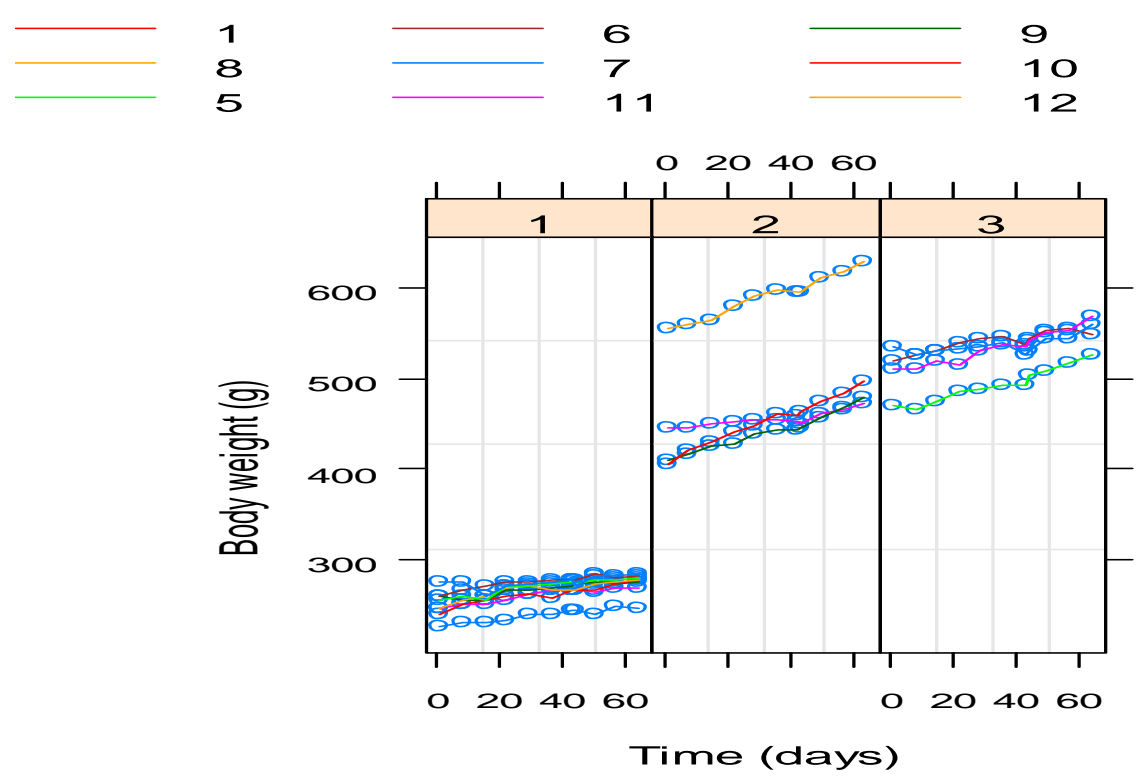


Fig.2 Dot-Plot of bodyweight of chicks measured over a period of 64 days. The chicks are divided into three groups on different diets

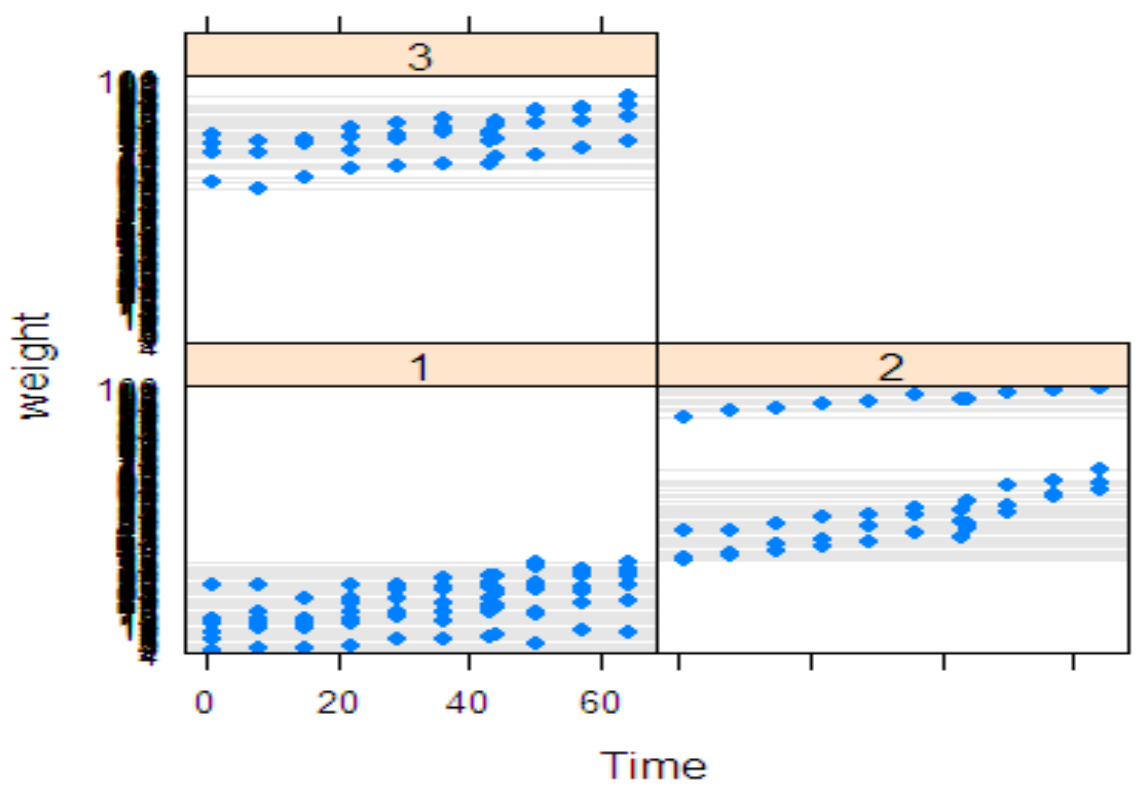

Fig.3 Residual plot corresponding to the fitted homocedastic model against number of chicks

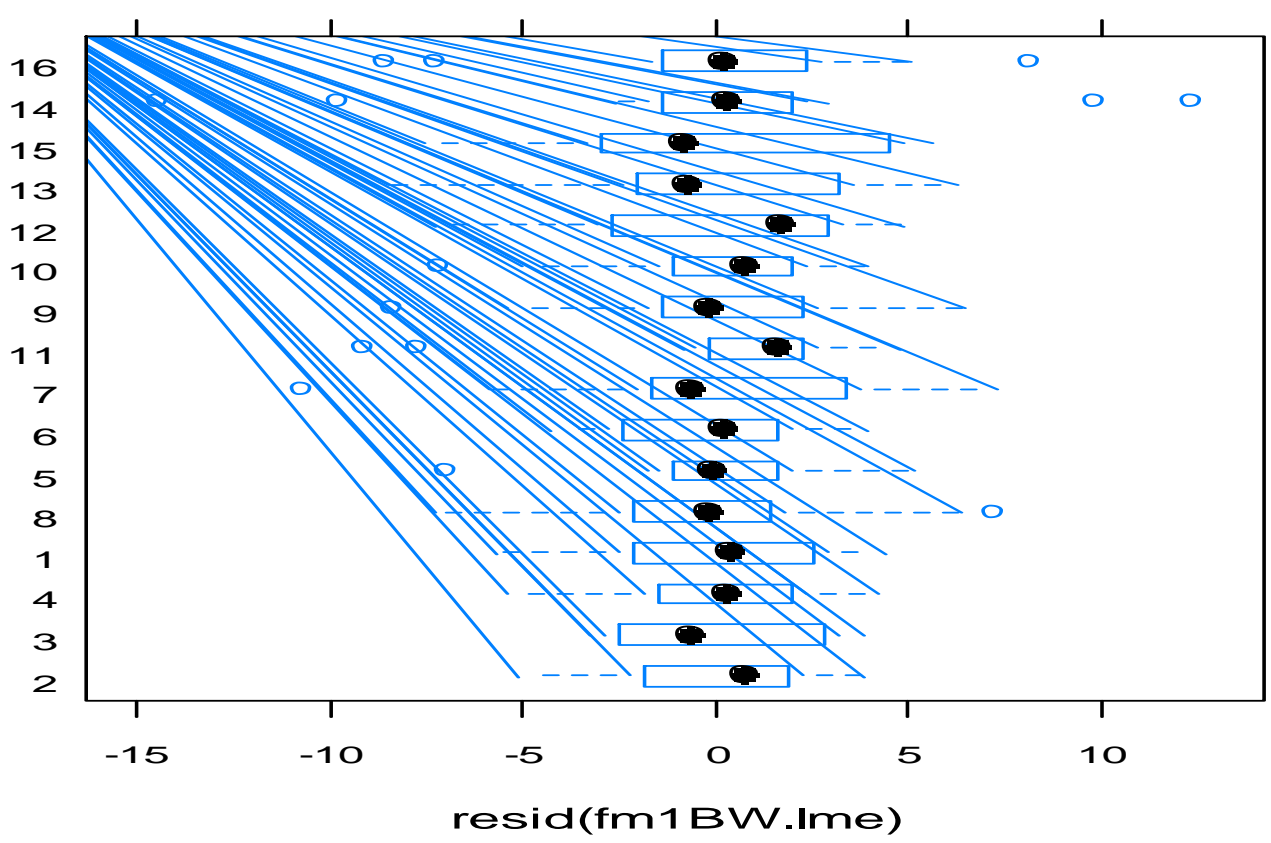


Fig.4 Plot of standardized residuals versus fitted values for the homoscedastic fitted model

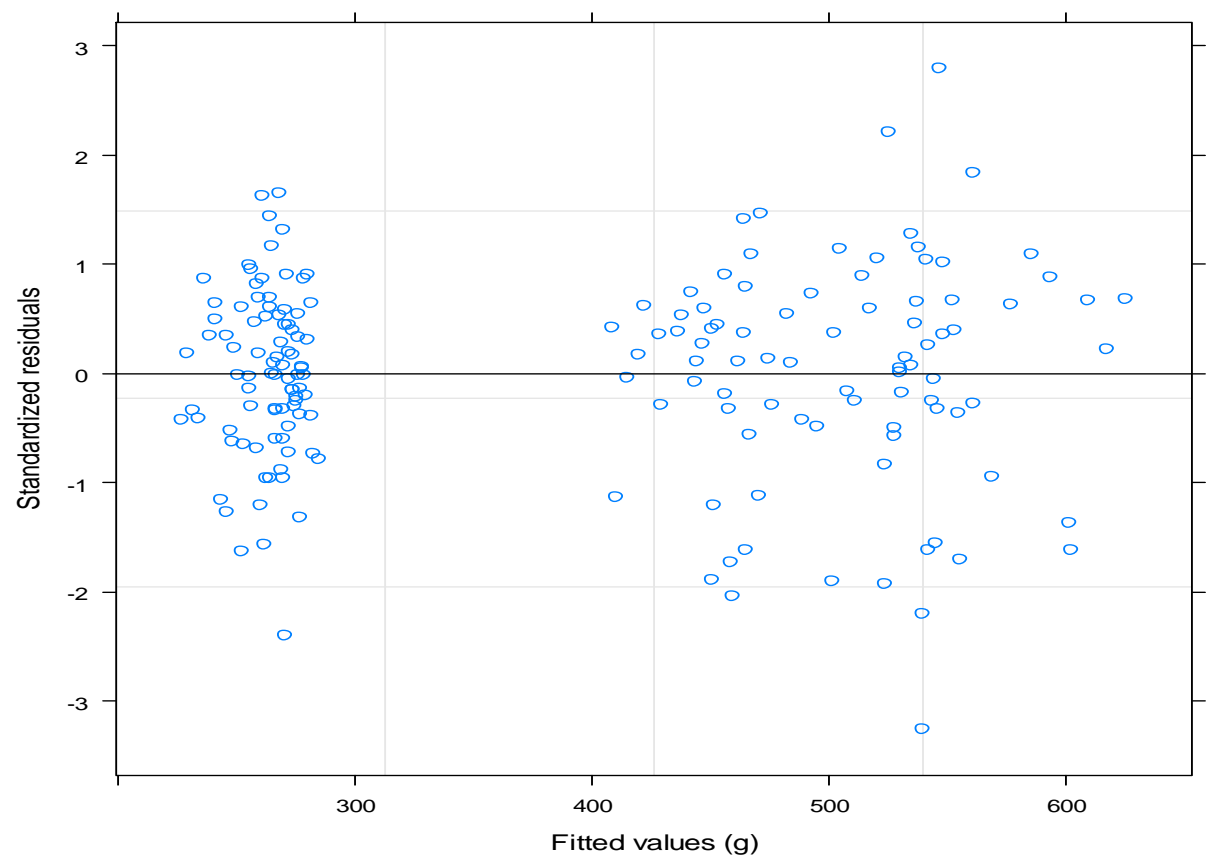

Fig.5 Residual plot corresponding to the fitted VarPower (heterocedastic) model against number of chicks

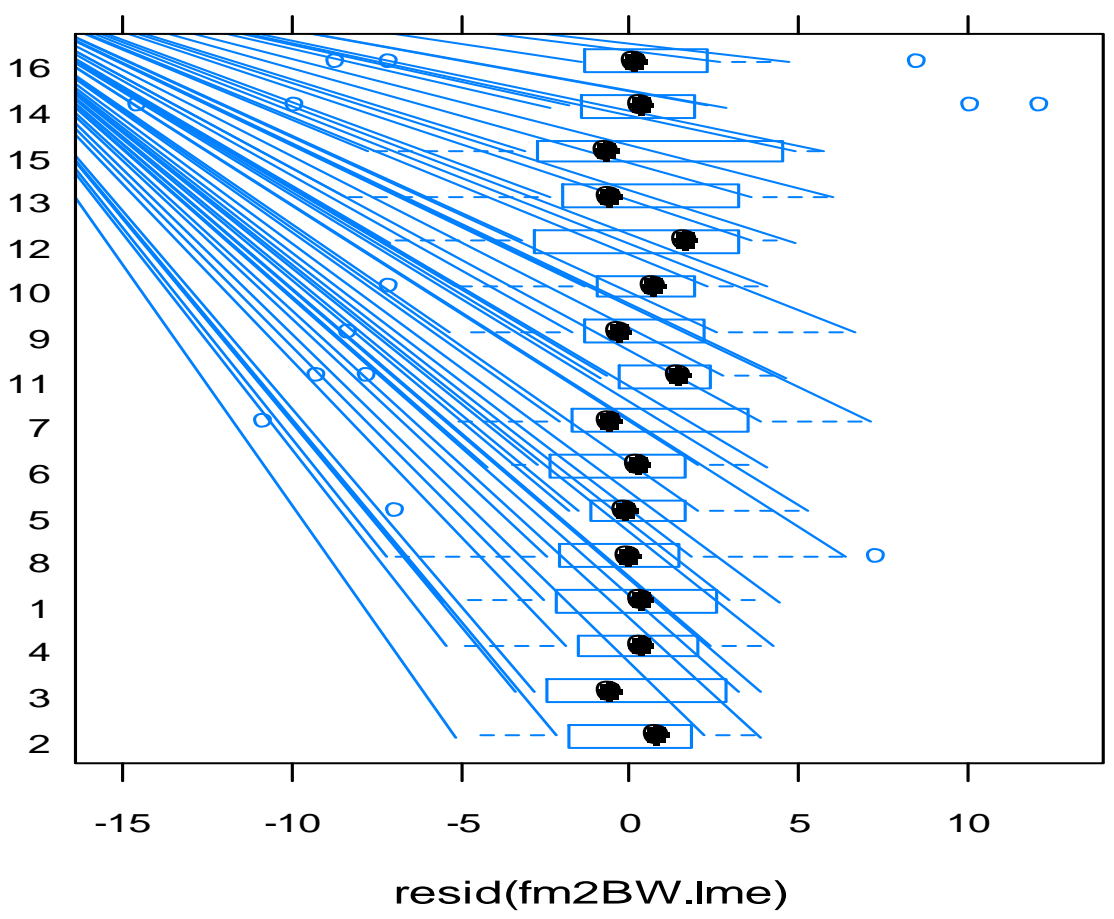


Fig.6 Plot of standardized residuals versus fitted values for the VarPower (heterocedastic) fitted model

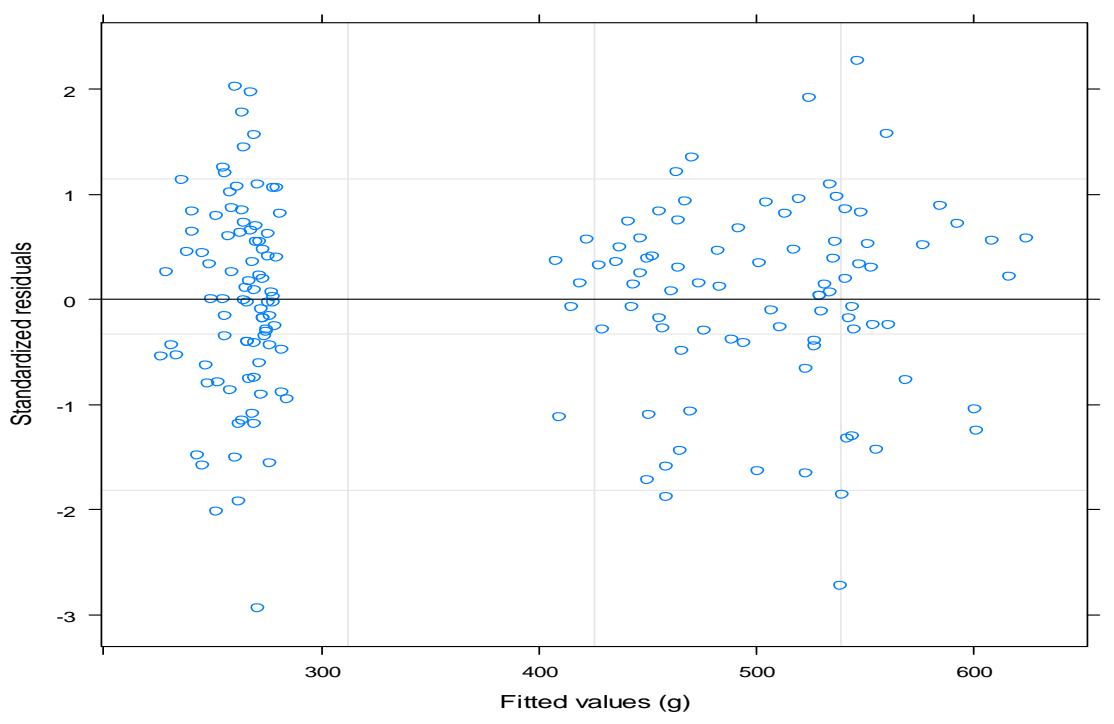

The approximate $95 \%$ confidence intervals for the fixed and random effects of the homocedastic mixed effects model are reported in Table 3 and 4 respectively

As can be seen from the values in the table the within group error assume a large value of 4.44 with a $95 \%$ interval of 3.95-4.98.

The box plots of residuals of the fitted homocedastic model for each of the chick is given in Figure 3. In each of the boxplot the dot in the center represents the mean value of residuals for a particular chick and the points outside the box represent the outlier residuals.

As can be seen from the Figure 3 the largest deviation in the residuals are associated with $13^{\text {th }}$ chick.

In order to check the adequacy of the homocedastic fitted model we make a plot of fitted values against standardized residuals which is given in Figure 4.

Figure 4, gives clear indication of withingroup heteroscedasticity. Because the fitted values are bounded away from zero, we can use the varPower variance function to model the heteroscedasticity. The variance model represented by varPower is

$$
\operatorname{Var}\left(e_{i j}\right)=\sigma^{2}\left|v_{i j}\right|^{2 \delta}
$$

Corresponding to the variance function

$$
g\left(v_{i j}\right)=\left|v_{i j}\right|^{\delta}
$$

Which is a power of the asolute value of variance covariate. The parameter $\delta$ is unrestricted (i.e., may take any value in the real line). The main arguments to varPower constructor are value and form, which specifies respectively an initial value of $\delta$, when this is allowed to vary in optimization and a one-sided formula with the variance covariate.

We simply used the update function to incorporate the heterocedasticity in the already fitted homocedastic model and name this model as varPower model. The results obtained are summarized in Table 5: Table 5 provides the values of different fixed effects 
and their interaction values along with their standard error, t-cal and p-value. From the table it is clear that there appears to be significant increase in growth rate associated with Diet2 (TimeDiet2) and a boarder line significant increase in growth rate for Diet3 (TimeDiet3). We can further assess the variability in the parameter estimates with the intervals method.

The approximate $95 \%$ confidence intervals for the fixed and random effects of the homocedastic mixed effects model are reported in Table 6 and Table 7, respectively.

As can be seen from the values in the table the within group error assume a smaller value of 0.18 with a $95 \%$ interval of $0.024-0.13$.

The box plots of residuals of the fitted heterocedastic model for each of the chick is given in Figure 5. A big dot in the center of each box plot represents the mean value of residuals for a particular chick and the dotted lines on both sides of box shows the range of the residuals. Also the points outside the box represent the outlier residuals.

As can be seen from the Figure 5 the largest deviation in the residuals are associated with $13^{\text {th }}$ chick Also smallest range of residuals is seen in the $14^{\text {th }}$ chick.

To check the adequacy of the heterocedastic fitted model we can make a plot of fitted values against standardized residuals which is given in Figure 6.

From the figure it is clear that the varPower variance function adequately represents the within group heteroscedasticity.

We can test the significance of the variance parameter in the var Power model using the anova method, which, as expected, strongly rejects the assumption of homoscedasticity (i.e., $\delta=0$ ). The results obtained by using the ANOVA method are summarised in Table 8.

AIC and BIC vales are the model selection criterion lesser the value of the AIC/BIC better is the model fit. Thus it can be concluded from the results in the table above that the var Power (heterocedastic) model is the better fit since its AIC/BIC is lowest also the likelihood ratio test is significant which supports our conclusion.

\section{References}

Bates, D., Maechler, M., and Bolker, B. (2011). mlmRev: Examples from Multilevel Modelling Software Review. $\mathrm{R}$ package version 1.0-1.

Bates, D., Maechler, M., and Bolker, B. (2012). MEMSS: Data sets from Mixed effects Models in $\mathrm{S}$. $\mathrm{R}$ package version 0.9-0.

Bates, D., Maechler, M., Bolker, B., and Walker, S. (2015). Fitting linear mixed effects models using lme4. Journal of Statistical Software, 67: 1\{48.

Davidian, M. and Giltinan, D.M. 1995. Nonlinear Models for Repeated Measurement Data, Chapman \& Hall, London.

Dempster, A.P., Laird, N.M. and Rubin, D.B. 1977. Maximum likelihood from incomplete data via the EM algorithm. Journal of the Royal Statistical Society, Ser. B 39: 1-22.

Gelman, A. and Pardoe, I. (2006). Bayesian measures of explained variance and pooling in multilevel (hierarchical) models. Technometrics, 48(2): $241\{251$.

Jiang, J. (1998). Asymptotic properties of the empirical BLUP and BLUE in mixed linear models. Statistica Sinica, 8(3): $861\{886$ 
Laird, N.M. and Ware, J.H. 1982. Randomeffects models for longitudinal data. Biometrics 38: 963-974.

Lindstrom, M.J. and Bates, D.M. 1988. Newton-Raphson and EM algorithms for linear mixed-effects models for repeated-measures data. Journal of the American Statistical Association 83: 1014-1022.

Longford, N.T. 1993. Random Coefficient Models, Oxford University Press, New York.

Loy, A. (2013). HLMdiag: Diagnostic tools for hierarchical (multilevel) linear models. $\mathrm{R}$ package version 0.2.2.
Pinheiro, J.C. and Bates, M.D. 2000. MixedEffects Models in $S$ and $S$ PLUS. Springer-Verlag New York.

Pinho, L.G.B., Nobre, S.J. and Freilas, S.M. 2012. On linear mixed models and their influence diagnostics applied to an actuarial problem. Chilean Journal of Statistics 3(1): 57-73.

R Core Team (2013). R: A Language and Environment for Statistical Computing. R Foundation for Statistical Computing, Vienna, Austria.

Thisted, R.A. 1988. Elements of Statistical Computing, Chapman \& Hall, London.

Wickham, H. (2009). ggplot2: elegant graphics for data analysis. Springer New York.

\section{How to cite this article:}

Nageena Nazir, S.A. Mir, Zahoor Ahmed and Iqbal Jeelani, M. 2018. Extension of Basic Linear Mixed Effect Model to Incorporate Heterocedasticity. Int.J.Curr.Microbiol.App.Sci. 7(06): 1061-1072. doi: https://doi.org/10.20546/ijcmas.2018.706.126 